\title{
Reflexões acerca da conceituação de saúde e construção de um conceito de saúde: implicações para os profissionais da saúde
}

\author{
Ana Cláudia Schuab Faria de Paula \\ Enfermeira (UFES). Mestre em Enfermagem pelo Programa de Pós-Graduação em Enfermagem da \\ Universidade Federal do Rio Grande (PPGEnf/FURG). Especialista em Saúde coletiva com ênfase em Saúde da \\ Família (UNIVC). Especialista em Enfermagem do Trabalho (FISIG). Enfermeira Assistencial Federal \\ (EBSERH/FURG) \\ $\triangle$ cacausfp.enf@hotmail.com

\section{Romario Daniel Jantara} \\ Enfermeiro. Mestrando em Enfermagem pelo Programa de Pós-Graduação em Enfermagem da \\ Universidade Federal do Rio Grande (PPGEnf/FURG).

\section{Daiane Porto Gautério Abreu} \\ Enfermeira. Especialista em Saúde da Família. Doutora em Enfermagem pelo Programa de Pós- \\ Graduação em Enfermagem da Universidade Federal do Rio Grande (PPGEnf/FURG). Docente do Programa de \\ Pós-Graduação em Enfermagem da Universidade Federal do Rio Grande (PPGEnf/FURG).

\section{Marlise Capa Verde Almeida de Mello} \\ Enfermeira. Doutora em Enfermagem pelo Programa de Pós-Graduação em Enfermagem da \\ Universidade Federal do Rio Grande (PPGEnf/FURG). Especialista em Enfermagem do Trabalho pela UNOPAR. \\ Enfermeira Técnica do Laboratório de Práticas em Enfermagem.
}

\section{Resumo:}

O debate sobre o conceito de saúde é extenso, envolvendo convergências e divergências recorrentes entre autores. Este estudo trata-se de um ensaio teórico-reflexivo que descreve a elaboração de um conceito de saúde por uma estudante de mestrado, embasado no plano de estudo da disciplina "Conceitos de saúde e seus nexos no trabalho da Enfermagem/Saúde", pertencente ao Curso de Mestrado em Enfermagem do programa de Pós-Graduação stricto sensu de uma Universidade Federal do extremo Sul do Brasil. o objetivo é estimular uma reflexão plural e edificadora no meio acadêmico e profissional sobre saúde, a partir da apresentação de uma construção conceitual fundamentada em diferentes saberes, teorias e nexos. As reflexões apresentadas ao longo deste estudo oportunizaram a construção de um conceito de saúde ampliado, compreendido como o estado subjetivo de bem-estar, satisfação, autonomia e funcionalidade, independente da dimensão, para realizar atividades propostas ou esperadas por um do indivíduo em relação à ele mesmo, frente à aspectos multidimensionais e multifatoriais intrínsecos/extrínsecos, considerando fatores limitantes não modificáveis, condições de vida/trabalho, e capacidade de adaptação e interação com o meio, transmudando assim o próprio ser. Considera-se que as discussões sobre saúde são infindáveis e seu entendimento constantemente ressignificado, seja de forma individual ou coletiva, social ou intelectual.

Palavras-chave: Saúde, Promoção da Saúde, Conceito de Saúde, Formação de Conceito. 


\title{
Reflections on the concept of health and the construction of a health concept: implications for health professionals
}

\begin{abstract}
:
The debate on the concept of health is extensive, involving convergences and recurring divergences between authors. This study is a theoretical-reflective essay prepared by a Nurse for the broad conceptual construction on health, based on the study plan of the discipline "Health concepts and their connections in the work of Nursing / Health", belonging to the Post- Stricto sensu graduation from a Federal University in the extreme south of Brazil. The objective is to stimulate a plural and edifying reflection in the academic and professional environment on health, based on the presentation of a conceptual construction based on different knowledge, theories and nexuses. The reflections presented throughout this study enabled the construction of an expanded health concept, understood as the subjective state of well-being, satisfaction, autonomy and functionality, regardless of the dimension, to carry out activities proposed or expected by one of the individual in relation to the himself, facing intrinsic / extrinsic multidimensional and multifactorial aspects, considering nonmodifiable limiting factors, living / working conditions, and the ability to adapt and interact with the environment, thus transmuting one's own being. Discussions about health are considered endless and their understanding is constantly reframed, whether individually or collectively, socially or intellectually.

Keywords: Health, Health Promotion, Health Concept, Concept Formation.

\section{Reflexiones sobre el concepto de salud y la construcción de un concepto de salud: implicaciones para los profesionales de la salud}

\section{Resumen:}

El debate sobre el concepto de salud es extenso, involucrando convergencias y divergencias recurrentes entre autores. El presente estudio es un ensayo teórico-reflexivo elaborado por una Enfermera para la construcción conceptual amplia en salud, a partir del plan de estudios de la disciplina "Conceptos de salud y sus conexiones en el trabajo de Enfermería / Salud", perteneciente a la Post- Graduado en estricto sensu de una Universidad Federal en el extremo sur de Brasil. El objetivo es estimular una reflexión plural y edificante en el ámbito académico y profesional sobre la salud, a partir de la presentación de una construcción conceptual basada en diferentes saberes, teorías y nexos. Las reflexiones presentadas a lo largo de este estudio permitieron construir un concepto de salud ampliado, entendido como el estado subjetivo de bienestar, satisfacción, autonomía y funcionalidad, independientemente de la dimensión, para realizar actividades propuestas o esperadas por uno de los individuos en relación con la él mismo, enfrentando aspectos intrínsecos/extrínsecos multidimensionales y multifactoriales, considerando factores limitantes no modificables, condiciones de vida/trabajo, y la capacidad de adaptarse e interactuar con el entorno, transmutando así. Las discusiones sobre salud se consideran interminables y su comprensión se replantea constantemente, ya sea individual o colectivamente, social o intelectualmente.

Palabras clave: Salud, Promoción de la Salud, Concepto de Salud, Formación de Conceptos.

\section{INTRODUÇÃO}

É crescente a preocupação mundial com questões relacionadas à saúde (BEZERRA; SORPRESO, 2016), assim, entender sobre com o que se preocupar é fundamental. Desde os 
primórdios da humanidade, o ser humano se questiona sobre a origem da vida; as razões da existência; e, o que significa ter saúde. Do mesmo modo, os profissionais de saúde, que utilizam seu trabalho como um meio para promoção da saúde e não apenas o para o tratamento de doenças, devem manter este essencial e existencial questionamento: 0 que é a saúde que se almeja alcançar? (SILVA; LINS; CASTRO, 2016).

Sabe-se que a saúde se associa intimamente com a qualidade de vida, sendo basilar para o desenvolvimento humano em diferentes nichos, seja pessoal, intelectual, biológico, econômico, histórico, ambiental ou social. Todavia, definir ou expressar de forma satisfatória o termo saúde e, sobretudo, utilizá-lo de forma atemporal e universal não é algo simples (CARRAPATO; CORREIA; GARCIA, 2017).

Apesar das diversidades e ausência de consenso, a busca pela formulação de um conceito operativo se faz necessária no trabalho em saúde. Os profissionais de saúde, incluindo o Enfermeiro, necessitam de um símbolo mental ou de uma noção abstrata retratada, para que possa conceber uma compreensão e emitir uma conclusão por meio de uma opinião manifesta que direcione, dentre muitos fatores, a tomada de decisões, a forma de abordagem, o delineamento de programas/ações, a prestação de assistência, a avaliação de resultados (MICHAELIS, 2016; PORTA, 2014; GALLO, 2008; CARRAPATO; CORREIA; GARCIA, 2017). Assim, o conceito de saúde reflete no aparecimento de novas formulações sobre o pensar e fazer sanitário e, por consequência, em propostas de mudanças dos modelos assistenciais em saúde (BEZERRA; SORPRESO, 2016).

No ano de 1945, Organização Mundial de Saúde (OMS) a elaborou um conceito de saúde, conceituando-a como "um estado de completo bem-estar físico, mental e social e não somente ausência de afecções e enfermidades" (OMS, 2006). Um sentido bastante utópico por visar uma completude inatingível, sobretudo no Brasil, um país historicamente marcado por profundas desigualdades sociais, econômicas, culturais e territoriais (GODOI, 2019). Somado a isto, através da Constituição Federal Brasileira de 1988, a saúde foi considerada um direito de todos e dever do Estado, devendo ser garantida mediante políticas socioeconômicas que visassem a redução de doenças, assim como pelo acesso universal e igualitário às ações e serviços de promoção, proteção e recuperação da saúde (BRASIL, 2016; GODOI, 2019). 
Todavia, diante da complexidade do significado de saúde, questiona-se se o Estado é capaz de assumir o dever garantidor deste direito, ou se deveria ser garantidor de meios facilitadores para que a saúde, pelo princípio da equidade, seja alcançada por todos. Setenta anos após o primeiro conceito de saúde ser apresentado pela OMS, a comunidade científica e acadêmica continua se indagando sua verossimidade (FERREIRA, 2014). Distintas tentativas estão sendo realizadas a fim de elaborar um conceito dinâmico e menos restritivo, reduzido ou engessado, que se afaste da antonímia de doença e se aproxime de uma visão ampliada, que considere as potencialidades e particulares da vida (SILVIA; LINS; CASTRO, 2016).

A compreensão de saúde tem alto grau de subjetividade e determinação histórica, a depender do momento, do referencial e dos valores atribuídos a cada situação, tratando-se de um estado transitório. Indivíduos podem expressar conceitos de saúde de forma cotidiana e irrefletida, através de comportamentos, como por exemplo, quando constroem ou definem estilos de vida a serem seguidos. Nestes momentos, a auto concepção de saúde se sobressai na forma de decisões e prioridades, relacionadas consciente ou inconscientemente, à determinadas concepções de saúde-cuidado (PRADO et al., 2016; COSTA, 2019).

Diante das possibilidades de compreensão sobre saúde, intelectuais divergem e convergem sobre suas concepções na busca de uma resposta concreta e universal. No entanto, independe do conceito atribuído ou da resposta dada às perguntas emergentes, ressalta-se a pertinência de abordagens e reflexões na busca pelo entendimento sobre saúde a fim de instrumentar o trabalho em saúde. Desse modo, este estudo tem por objetivo estimular uma reflexão plural e edificadora no meio acadêmico e profissional sobre saúde, a partir da apresentação de uma construção conceitual fundamentada em diferentes saberes, teorias e nexos.

\section{METODOLOGIA}

Este estudo compreende a construção conceitual sobre saúde e suscita reflexões a partir de um ensaio teórico-reflexivo, uma vez que elementos teóricos foram analisados e interpretados, para sistematizar um robusto corpo de informações e conhecimento 
(MENEZES et al., 2019). Ele engloba formulações teóricas de saúde e adoecimento; processos de codificação das formulações no trabalho em saúde; saberes tecnológicos orientadores do trabalho e seus nexos nas estruturas conceituais de saúde e de adoecimento de seres humanos em diferentes espaços socioambientais; o trabalho em saúde, suas inter-relações e possibilidades de intervenção para a adequação da vida humana e não humana.

O estudo foi realizado por uma enfermeira, estudante de mestrado. O estudo emergiu como fruto da disciplina "conceitos de saúde e seus nexos no trabalho da Enfermagem/Saúde", pertencente Curso de Mestrado em Enfermagem do Programa de PósGraduação Stricto Sensu de uma Universidade Federal do extremo Sul do Brasil, realizada em dez encontros presenciais, durante o primeiro semestre do ano de 2019, por meio de um cronograma pré-estabelecido.

No primeiro encontro a estudante de mestrado elaborou um conceito inicial de saúde que serviu de pilar para a construção do seu conceito final ampliado, sendo mantido os aspectos primários de sua definição ao longo de todo o processo. O conceito inicial surgiu da transcrição de ideias, conhecimentos e experiências prévias individuais, sendo gradualmente expandido e ressignificado ao longo de cada encontro, mediante as leituras curriculares, extracurriculares, e discussões guiadas por duas docentes, enfermeiras, doutoras em Enfermagem, em conjunto com os demais estudantes da turma por meio de diferentes métodos (construção de mapas conceituais, discussão dialógica grupal, construção de resumos, apresentações em multimídia e dinâmicas grupais). A construção do conceito e seu aprimoramento foi um exercício individual.

O programa disciplinar incluiu os autores e obras descritas no quadro 1. 
Quadro 1 - Referências indicadas para leitura na disciplina e utilizadas na construção do conceito de saúde

\begin{tabular}{|l|l|}
\hline \multicolumn{1}{|c|}{ AUTORES E ANO } & \multicolumn{1}{c|}{ TÍTULO DAS OBRAS } \\
\hline JJ Camargo (2015) & Do que você precisa para ser feliz \\
\hline Georges Canguilhem (2012) & O Normal e o Patológico \\
\hline Karl Marx (2017) & O Capital \\
\hline Guattari e Rolnik (2005) & Micropolítica - cartografias do desejo \\
\hline Enrique Leff (2012) & $\begin{array}{l}\text { Saber Ambiental: } \\
\text { racionalidade, complexidade, poder; }\end{array}$ \\
\hline Ministério da Saúde (Brasil, 2008) & $\begin{array}{l}\text { As causas sociais da iniquidade em saúde no } \\
\text { Brasil. }\end{array}$ \\
\hline Janaína Ávila (2012) & $\begin{array}{l}\text { Funcionalidade } \\
\text { institucionalizada com risco de queda: } \\
\text { proposta de cuidado de enfermagem }\end{array}$ \\
\hline
\end{tabular}

Fonte: Os autores, 2021.

As leituras recomendadas foram discutidas em grupo por acreditar que a fala e os conceitos são mediadores ideológicos entre o indivíduo e o grupo. Acredita-se não haver um sentido único para a apreensão do conhecimento, assim, a interação dialógica é um importante meio para se estabelecer a comunicação e a construção de ideias, através do somatório das contribuições e conflitos, sendo a interação pautada pelos diálogos descentralizada dos sujeitos (SILVA; BORGES, 2017). A metodologia da interação dialógica ampliou a percepção e as reflexões sobre saúde, permitindo a negociação, a cooperação, o respeito às individualidades; e a construção de ideias e sentidos para o que se define como saúde. 


\section{RESULTADO}

O conceito primário ou inicial de saúde, definido como o estado de bem-estar, satisfação e capacidade funcional particular, de cada indivíduo, para realizar as atividades por ele propostas ou esperadas, diante dos fatores limitantes não modificáveis, foi ampliado ao longo do processo construtivo e entendido, ao final deste estudo, como o estado subjetivo de bem-estar, satisfação, autonomia e funcionalidade, independente da dimensão, para realizar atividades propostas ou esperadas por um do indivíduo em relação à ele mesmo, frente à aspectos multidimensionais e multifatoriais intrínsecos/extrínsecos, considerando fatores limitantes não modificáveis, condições de vida/trabalho, e capacidade de adaptação e interação com o meio, transmudando assim o próprio ser.

\section{DISCUSSÃO}

Os autores e seus estudos analisados e discutidos para a constituição deste artigo apresentam diversas abordagens que estimularam a construção de novos saberes relacionados à saúde e a doença, que nos lançam em novos olhares possíveis acerca destes dois fenômenos presentes na vida em sociedade. Nesse sentido, são apresentados os principais estudiosos que fundamentaram a compreensão e construção do conceito de saúde elaborado, bem como, são explicitadas "palavras-chaves" retiradas de cada leitura para compor o conceito de saúde formulado.

Destaca-se, os estudos realizados por Guattari, em conjunto com Rolnik (GUATTARRI; ROLNIK, 2005). Este título aborda a questão da subjetividade coletiva e individual, e a singularidade dos sujeitos. Para estes autores, a subjetividade oscila conforme o modo de viver dos indivíduos, podendo se submeter à subjetividade tal como a recebe, ou então estabelecer uma relação de expressão e de criação, apropriando-se dos componentes da subjetividade coletiva e produzindo o processo de singularização (GUATTARRI; ROLNIK, 2005).

O processo de singularização da subjetividade se faz emprestando, associando e aglomerando dimensões de diferentes espécies, assim, o que vai caracterizar um processo de 
singularização é que ele, por si próprio, seja automodelador. Essa capacidade de automodelação vai permitir um mínimo de possibilidade de criação, não sendo rígida as formas de relação, possibilitando preservar o caráter de autonomia (GUATTARRI; ROLNIK, 2005).

Ressalta-se que, as mutações da subjetividade, não funcionam apenas no registro das ideologias, mas no próprio coração dos indivíduos, em sua maneira de perceber o mundo e de se articular com o meio, isto é, o modo como se percebe o meio e o modo como cada um se afeta pelo meio reflete de forma dinâmica, frequente e cíclica nas construções particulares. A produção de subjetividade constitui a matéria prima de toda e qualquer produção (GUATTARRI; ROLNIK, 2005). Desta maneira, ao evidenciar a saúde como um processo de produção, observa-se que, seu significado está diretamente relacionado à produção da subjetividade.

Portanto, faz sentido crer que essa produção de subjetividade pode interferir no entendimento de saúde das pessoas e na tomada de decisão das mesmas sobre sua saúde, ou seja, o indivíduo interpreta, julga e modela sua saúde em conformidade com o que está estabelecido em âmbito extrínseco ou social, modelando comportamentos, sensibilidade, percepções, memórias, relações sociais, e sexuais, entre outras (GUATTARRI; ROLNIK, 2005). Assim, incorporou-se ao conceito de saúde o termo "subjetivo", considerando a influência da subjetividade individual e coletiva na saúde dos indivíduos.

Leff (2012), reforça o conceito de subjetividade trazido por Guattari e Rolnik (2005). Leff (2012), defende que existem diferentes vias para se caracterizar a qualidade de vida das populações e dos indivíduos. Para ele, a saúde é influenciada pelo ambiente, contexto social, histórico e cultural em que o indivíduo está inserido, sendo estes, determinantes da qualidade de vida, os quais estruturam as necessidades pessoais e os meios para satisfazê-las. Este estudioso, se utiliza de uma nova vertente de articulação, atrelando a satisfação à subjetividade individual para alcançar o que se considera bem-estar e qualidade de vida.

Ainda para este autor, a qualidade de vida se estabelece através de um processo de reapropriação das condições de vida da população em relação com suas necessidades e valores subjetivos, sendo imensuráveis as necessidades básicas e as aspirações culturais que 
definem a qualidade de vida dentro das diferentes formas de desenvolvimento. Isto leva à aceitação do relativismo implícito na reivindicação da autogestão ou autonomia na gestão do que acredita ser qualidade de vida (LEFF, 2012). Assim, o termo "satisfação" presente no conceito formulado foi relacionado à essa leitura, por reforçar a influência da subjetividade já tratada por Guatarri e Rolnik (2005) e introduzir um dos aspectos multidimensionais do indivíduo, a cultura, que também é abordada por Ávila (2012), além de trazer a questão da saúde estar diretamente relacionada com a qualidade de vida e bem-estar dos sujeitos.

Ávila (2012), considera além da cultura, outros aspectos multidimensionais do indivíduo, como o contexto familiar, comunitário, emocional e espiritual, como preconizado pela Classificação Internacional de Funcionalidade, Incapacidade e Saúde (WORLD HEALTH ORGAZINATION, 2007), acreditando-se que através dessa perspectiva, pode-se promover avanços e criar estratégias para a manutenção da autonomia e prevenção de agravos, corroborando assim para a preservação da saúde. A saúde para ela também é afetada por fatores fisiológicos, cognitivos, físicos, ambientais e físico-geográficos, denominados fatores intrínsecos e extrínsecos (ÁVILA, 2012). Desta autora, emergiram os temas “autonomia”, "aspectos multidimensionais" e "interferência multifatorial intrínseca e extrínseca", que foram acrescentados ao conceito inicial.

Sorre (1943) trata da funcionalidade, demonstrando que o homem considerado coletivamente está à procura de seus "ideais funcionais", isto é, dos valores de cada um dos elementos do ambiente para os quais se realiza melhor uma função determinada. Para este teórico, as constantes fisiológicas não são constantes no sentido absoluto do termo, havendo uma margem na qual se introduz a capacidade de adaptação funcional do grupo ou da espécie, para cada função ou para o conjunto das funções. Diante disso, foi indexado a "capacidade de se adaptar" ao conceito inicial, considerando os aspectos relativos à necessidade de adaptação.

A autonomia do indivíduo não é apenas física, mas também psíquica, Canguilhem, perpetrou a reflexão para além do processo de singularização de Guattari e Rolnik (2005) ao tratar da norma supra individual, e associá-la ao normal e ao patológico, à doença, à saúde, e à cura. A saúde se trata também de um estado valorizado e estimado pelas pessoas, uma vez que Canguilhem (2012), discute que a doença, na norma da sociedade, carrega a conotação de 
nocividade e desvalorização, inferindo ainda que as constantes patológicas têm valor repulsivo e estritamente conservador.

Em sua perspectiva, a saúde não pode ser reduzida e limitada à aspectos quantitativos da variação dos fenômenos fisiológicos. Ao trabalhar as inter-relações sofridas e refletidas pelos seres, Canguilhem tornou possível perceber que ao próprio ser vivo cabe a responsabilidade de distinguir o ponto em que começa a doença. Ele afirma, assim como Laugier (1937), que uma média obtida estatisticamente não permite dizer se o indivíduo, presente diante de nós, é normal ou não; que é impossível determinar o ser doente quanto ao conteúdo por se tratar de uma norma supraindividual.

Esta ideia é realçada por Sigerist (1932), ao expor que o indivíduo não deve se limitar a estabelecer a comparação dos seres como uma norma resultante da média, e sim, na medida do possível, considerando as condições e particularidades do ser examinado. Portanto, a pessoa não é doente apenas em relação aos outros, mas em relação a si mesma. Apesar do próprio da doença ser interromper o curso de algo ou ser crítica, mesmo quando se torna crônica, há sempre um passado do qual o paciente ou aqueles que o cercam guardam certa nostalgia e que irá diferir na forma como cada um irá enfrentar a doença (CANGUILHEM, 2012). A partir da abordagem destes autores, foi incluída a síntese "ele em relação a ele mesmo", reforçando o entendimento do próprio indivíduo sobre a particularidade do que se considera ter saúde.

A forma como cada ser enfrenta os obstáculos, absorve as notícias e lida com as perdas, reflete a gratidão, demonstra empatia ou sustenta a espiritualidade. Camargo (2015), leva à reflexão de que saúde é um conceito amplo que envolve uma gama de processos individuais a cada pessoa. $O$ autor motiva o pensamento de que a realidade em que se vive é composta por uma complexidade de relações, onde se experimenta diversos sentimentos e momentos, que moldam cada indivíduo no cotidiano, tornando-os seres únicos, não fisicamente, mas sim, na capacidade de expressar emoções e sentimentos próprios (CAMARGO, 2015).

Para Camargo (2015), a superação pode ser encontrada em pequenas coisas, como voltar a respirar normalmente ou tomar um banho, e o sofrimento crônico pode fazer com que o indivíduo aprenda a alegria das pequenas coisas quando as grandes coisas se tornam inalcançáveis; não sendo possível dimensionar a representação individual dos eventos ou dos 
seus significados e sensações. Com isso, "independente da dimensão" foi adicionado ao conceito, considerando que o que é significativo ou representativo para um indivíduo pode ser percebido de forma contraria ou distinta pelo outro ou até por ele mesmo, a depender do momento e das condições de vida particulares.

As condições de vida e também as condições de trabalho são elementos pertencentes ao meio em que o sujeito se insere, sendo considerados determinantes sociais para a saúde. 0 relatório final da Comissão Nacional sobre Determinantes Sociais da Saúde (CNDSS) aborda que as condições materiais de vida e trabalho dos indivíduos e grupos, assim como sua vulnerabilidade aos impactos ambientais estão fortemente influenciadas pela posição social que ocupam e que pode ser definida por meio de uma série de variáveis, dentre elas a renda, a escolaridade e o gênero (BRASIL, 2008).

Estas condições de vida e trabalho e seus efeitos psicossociais, constituem um dos principais mediadores, através dos quais a estratificação socioeconômica influencia a situação de saúde dos indivíduos e grupos, bem como os diferenciais observados entre eles. A alimentação, cujo acesso e qualidade estão claramente influenciados por fatores socioeconômicos, comportamentais e culturais, constitui um dos determinantes sociais mais relevantes para a saúde, interferindo diretamente, por exemplo, na disposição energética para realização das atividades diárias e no estado nutricional (BRASIL, 2008). A partir desta leitura "condições de vida e trabalho" se fez fundamental ao conceito.

O processo de trabalho, o meio para o trabalho e o meio ambiente, são abordados de forma recorrente pela literatura de Karl Marx. Para ele, participam do processo de trabalho, o homem e a natureza, processo em que, o ser humano, através de sua ação, impulsiona, regula e controla o intercâmbio material ao interagir com a natureza. De certo modo, a saúde do homem depende dos processos desenvolvidos por este e de como se dão estes processos, assim, não é equivocado afirmar que a saúde depende da relação do homem com a natureza, uma vez que todos os processos resultantes do trabalho, são decorrentes desta interrelação influente no estado de saúde do indivíduo (MARX, 2017).

Colocando-se em movimento as forças naturais do corpo, braços e perna, cabeça e mão, a fim de se apropriar dos recursos naturais, imprime-se força útil à vida humana. Assim, o homem produz algo ao interagir com o meio, independentemente do que se produz, desde 
que se produza o que se propôs a produzir, que alcance o que se propôs à alcançar ou que tenha valor, mesmo que individual, o resultado da interação proposta (MARX, 2017). Dessa forma, apõe-se ao conceito, a partir desta ciência, o ato de "interagir com o meio natural, modificando assim o próprio ser", entendendo-se que, a saúde está relacionada com o meio em que o homem está inserido, onde ele é capaz de modificá-lo, e ao mesmo tempo, modificar a si próprio.

\section{CONSIDERAÇÕES FINAIS}

Refletir sobre os aspectos e processos relativos à saúde e seu significado, de forma mais subjetiva e menos objetiva é fundamental para o autoconhecimento, reconhecimento e melhoria da qualidade de vida. Para além do indivíduo, entender a significação de saúde expressa pelos sujeitos é uma tarefa complexa e primordial, especialmente aos profissionais de saúde, responsáveis pelo cuidado à vida humana.

Entende-se que o profissional, necessita reconhecer a compreensão de saúde expressa pelos clientes/família/comunidades/grupos/populações, o que faz parte do diagnóstico de saúde e da reorientação das práticas assistenciais dos serviços de saúde. O entendimento do conceito direcionado de saúde, torna-se um processo facilitador do trabalho, capaz de potencializar a capacidade de formular e implementar intervenções de forma clara, particular e genuína e excitar o alcance de resultados satisfatórios.

Os desequilíbrios capazes de afetar o estado considerável saudável, possuem etiologia em problemáticas muito variadas, e a reação aos considerados problemas de manifesta de forma não previsível, aproximando-se do previsível quando se possui um conhecimento sobre todo o contexto envolvido. Todavia, a compreensão do ser existencial é difícil inclusive para o próprio ser, que vivencia uma luta diária rumo à evolução, na busca de sabedoria para lidar com as adversidades sem se afetar negativamente sobremaneira e crescer em algum aspecto da vida como pessoa. Com isso, os padrões de saúde não deveriam ser universais e rígidos, mas sim flexíveis, moldáveis e adaptáveis. 
O conceito deve permitir esta metamorfose e fluidez, pois o significado de saúde pode cambiar inclusive para um único indivíduo no período do amanhecer ao anoitecer, e os parâmetros não possuem medida, cabe a cada um estabelecer seus próprios parâmetros. Acredita-se que o entendimento do conceito ampliado de saúde e dos seus significados particulares, é crucial para a prestação de uma assistência humanizada, no incentivo do cliente na auto busca de uma vida com saúde, e na compreensão de que ninguém melhor que o próprio ser para absorver suas necessidades e trabalha-las com hábitos e visões positivistas dentro dos próprios padrões. Com isso, este estudo contribui para novas reflexões, discussões e construções sobre saúde, que são infindáveis e devem ser constantemente ressignificadas.

\section{REFERÊNCIAS}

AVILA, A. J. Funcionalidade da pessoa idosa institucionalizada com risco de queda: proposta de cuidado de enfermagem. (Dissertação). Mestrado em Enfermagem. Universidade Federal de Rio Grande. Rio Grande, 2012. Disponível em:<http://repositorio.furg.br/handle/1/3517>. Acesso em: 12 jul, 2019.

BEZERRA, M.P; SORPRESO, I.C.E. Conceitos de saúde e movimentos de promoção da saúde em busca da reorientação de práticas Conceptsandmovements in healthpromotiontoguideeducationalpracticesitalla. J Hum Growth. v.26, n.1, p. 11-16, Dez. 2016. Disponivel em:<http://ciscacongresso.com.br/wp-content/uploads/2016/04/Portugu\%C3\%AAs261-Art.-1.pdf>. Acesso em: 02 fev 2020. DOI: http://dx.doi.org/10.7322/jhgd.113709

BRASIL. Comissão Nacional Sobre os Determinantes Sociais da Saúde (CNDSS). As causas sociais das iniquidades em saúde no Brasil: Relatório final. Rio de Janeiro. Editora Fiocruz, 2008.

BRASIL. Ministério da Saúde. Constituição da República Federativa do Brasil de 1988. Brasília, DF: Presidência da República, Brasília, 2016. Disponível em:<http://www.planalto.gov.br/ccivil_03/constituicao/constituicao.htm>. Acesso em: 08 jul 2019.

BRASIL. Ministério da Saúde. Oitava Conferência Nacional de Saúde-Relatório Final. Brasília,1986. Disponível em:< http://conselho.saude.gov.br/biblioteca/Relatorios/relatorio_8.pdf> . Acesso em 28 Jun. 2019.

CAMARGO, J.J. Do que você precisa para ser feliz? 2015. 240 p.

CANGUILHEM, G. O normal e o patológico. (Segunda Parte: Existem Ciências do Normal e do Patológico? I Introdução ao Problema; II Exame crítico de alguns conceitos: do normal, da anomalia e da doença, do normal ao experimental; III Norma e Média). $7^{a}$ ed. Rio de Janeiro; Forense-Universitária, 2012.

COSTA, N.C.G. O corpo ideal e a cultura fitness: configurações de um estilo de vida. Tese (Doutorado). Programa de pós graduação em cultura contemporânea. Universidade Federal de Mato Grosso. Cuiabá, 2019. 190f. Disponível em: <http://ri.ufmt.br/bitstream/1/1963/1/TESE_2019_Neuza\%20Cristina\%20Gomes\%20da\%20Costa.pdf>. Acesso em 03 mar 2020.

CARRAPATO, P; CORREIA, P; GARCIA, B. Health determinants in Brasil: searching for healthequity. Saúde Soc. São Paulo, v.26, n.3, p.676-689, 2017. Disponível em:<https://scielosp.org/article/sausoc/2017.v26n3/676-689/pt/>. Acesso em: 05 jun 2020. DOI 10.1590/S0104-12902017170304.

FERREIRA, C.F. Guia de Estudos: Organização Mundial da Saúde (OMS) 2014.Disponível em: < https://sinus.org.br/2014/wp-content/uploads/2013/11/OMS-Guia-Online.pdf> . Acesso em: 10 abr. 2009. 
Reflexões acerca da conceituação de saúde e construção de um

conceito de saúde: implicações para os profissionais da saúde

GALLO, E. D. A. Integração regional da saúde na América do Sul: desafios e prioridades. In: SOUZA, Maria de Lourdes de et al. A saúde e a inclusão social nas fronteiras. 2008. P. 63-92.

GUATTARI, F; ROLNIK, S. Micropolítica - cartografias do desejo. 7ª ed. Revisitada. Petrópolis: Editora Vozes; 2005.

LAUGIER (H.). O homem normal. Em: enciclopédia francesa. IV, 1937.

LEFF, Enrique. Saber Ambiental - Sustentabilidade, Racionalidade, Complexidade, Poder. Ed. VOZES. Ed 9. Petrópolis. RJ: Vozes, 2012.

MARX, K. o capital. Crítica da economia política. 31ª Ed. São Paulo: Difel. Liv. I, v.1, 2017. Capítulo 5 - Processo de Trabalho e Processo de produzir mais-valia, p. 211-223.

MENEZES, A. H. N.; DUARTE, F. R.; CARVALHO, L. O. R.; SOUZA T. E. S. Metodologia científica: teoria e aplicação na educação a distância. Universidade Federal do Vale do São Francisco, Petrolina-PE, 2019. Disponível em: <https://portais.univasf.edu.br/dacc/noticias/livro-univasf/metodologia-cientifica-teoria-e-aplicacao-naeducacao-a-distancia.pdf>. Acesso em: 22 mar 2020.

MICHAELIS. Dicionário Escolar: Língua Portuguesa. Nova ortografia conforme o acordo ortográfico da língua portuguesa. São Paulo, Editora Melhoramentos. n.4, 992f, 2016.

ORGANIZAÇÃO MUNDIAL DA SAÚDE (OMS). Constituição da Organização Mundial da Saúde. Documentos básicos, suplemento da $\quad 45^{\mathrm{a}}$ edição, outubro de $2006 . \quad$ Disponível em: <https://www.who.int/governance/eb/who_constitution_sp.pdf.>. Acesso em: 22 mar 2020.

PORTA, M. ED. A dictionaryofepidemiology. 6th ed.; 2014.

PRADO, S. D; AMPARO-SANTOS, L; SILVA, L. F. D; ARNAIZ, M. G; BOSI, M. L. M. Estudos socioculturais em alimentação e saúde: saberes em rede, vol. 5. Estudos socioculturais em alimentação e saúde: saberes em rede. Rio de Janeiro: EDUERJ, Universidade do Estado do Rio de Janeiro, 2016. Sabor metrópole series, vol. 5. Disponível em: <https://static.scielo.org/scielobooks/37nz2/pdf/prado-9788575114568.pdf>. Acesso em: 22 mar 2020.

SILVA, C.C; BORGES, F.T. Análise Temática Dialógica como método de análise de dados verbais em pesquisas qualitativas. Linhas Críticas, Brasília, DF, v.23, n.51, p.245-267, jun. 2017 a set. 2017.Disponível em: <https://www.redalyc.org/pdf/1935/193554180002.pdf>. Acesso em: 22 mar 2020.

ROZA, M. Uma relação entre conceito de saúde, normatividade e biopolitica. In: BAGRICHEVSKY, M.; ESTEVÃO, A. Saúde Coletiva: dialogando sobre interfaces temáticas. Ilhéus- BA: Editus, 2015. P. 91-119.

SIGERIST, H.-E. Introduction à lamédecine. Trad. fr. Paris: Payot, 1932.

SILVA, E.S; LINS, G.A; CASTRO, E.M.N.F. Historicidade e olhares sobre o processo saúde-doença: uma nova percepção. Revista SUSTINERE, Rio de Janeiro, v. 4, n. 2, p. 171-186, jul-dez, 2016. Disponível em:< https://doi.org/10.12957/sustinere.2016.25976>. Acesso em: 05 fev 2020.

SORRE, M. Os fundamentos biológicos da geografia humana. Paris: Colin, 1943.

WORLD HEALTH ORGANIZATION. International Classification of Functioning, Disability, and Health: Children \& Youth Version: ICF-CY. World Health Organization, 2007.

(c)) EY

Este trabalho está licenciado com uma Licença Creative Commons - Atribuição 4.0 Internacional. 